International Journal of Engineering \& Technology, $10(1)(2021) 38-45$
International Journal of Engineering \& Technology
SPC
Website: www.sciencepubco.com/index.php/IJET
Research paper

\title{
Thermo-fluidic transport of electromagnetohydrodynamic flow of sodium alginate based Casson nano fluid passing through a porous microtube under the effect of streaming potential
}

\author{
Motahar $\operatorname{Reza}^{1}$ *, Amalendu Rana ${ }^{1}$ \\ ${ }^{1}$ Department of Mathematics, School of Science, GITAM Deemed to be University, Hyderabad-502329, India \\ *Corresponding author E-mail: motaharreza90@gmail.com
}

\begin{abstract}
Thermal transport characteristics of Casson nanofluid through a porous microtube is analyzed under the effect of streaming potential and constant pressure gradient with electrokinetic effect associated with applied magnetic field. An analytical solution of the velocity and temperature distribution of Casson-nano fluid through the porous microtube related to combining effects of electromagnetohydrodynamics forces under the effect of streaming potential have been obtained. The significant influences of various non-dimensional parameters on velocity and temperature profiles are discussed in this study. Also, it is revealed the impact of nano particles on flow transport and heat transfer phenomenon. Furthermore, the Nusselt number is calculated analytically. The variations of pertinent parameters such as Hartmann number, Darcy number,Casson parameter, volume friction parameter of nanoparticles, joule heating parameter are delineated graphically and discussed in details.
\end{abstract}

Keywords: Electromagnetohydrodynamic Flow; Microtube; Porous Medium; Casson Fluid; Nano Fluid.

\section{Introduction}

To improve more advance microfluidic devices, microfluidic technology has been undertaken in so many fields such as bio chemical analysis instruments, micro-heat exchanger, biomedical systems, lab-on-chip devices, micropumps, micro-turbines etc [1,2]. There are number of researchers who have investigated the hydrodynamic and thermo-fluidic transport of the electroosmotic flow through a microchannel. Further, the measurements of streaming potential is also investigated by so many researchers. Heat transfer of a nano-fluid flow through a microchannel with trigular/rectangular ribs is studied by Behnampour et al [3]. Based on the new KKL (Koo-Kleinstreuer-Li) model Li and Kleinstreuer [4] investigated the thermal performance of nanofluid flow through a trapezoidal microchannel which is induced by the Brownian micro-mixing motion. Yang et al [5] represented the electroosmotic flow and streaming potential through a heterogeneous microtube with nonuniform zeta potentials the discussed about the streaming potential and the flow phenomenon due to electroosmosis. An analytical solution of streaming potential and the periodical electro viscous effects in a pressure driven flow through a uniform microchannel is done by Gong et al [6]. An experimental investigation is provided by Lu et al [7] on the flow field effect on the EDL of the microchannel with the streaming potential.

The previous studies are focused only on the electroosmotic flow. Recently there are so many analytical and experimental investigations are going on the electromagnetohydrodynamic (EMHD) flows to developed EMHD micropumps. The flow control phenomenon in a microchannel with the combined influence of electromagnetohydrodynamic effects is studied by Chakraborty and Paul [8]. Further thermal characteristics of a EMHD flow through microchannel under constant wall heat flux conditions is analysed by Chakraborty et al. [9]. Reza and Rana [10] investigated an analytical exact solution of EMHD flow and heat transfer of Casson fluid through microchannel with viscous dissipation and joule heating effect. Buren et al [11] studied EMHD flow through a micro parallel channel with corrugated wall by Perturbation technique. Very recently EMHD effects on porous microchannel with corrugated wall of a non-Newtonian second grade fluid investigated by Rashid et al [12] by applying the perturbation technique. An comprehensive analysis of heat transfer of nano-fluid through a microtube under the streaming potential effects is done by Zhao et al [13].

Sodium alginate is one kind of natural polymers which is the sodium salt of alginic acid. It is extracted from the cell walls of brown algae. It is used widely in various industries including food manufacturing, textile printing, cosmetics and pharmaceuticals. The applications of sodium alginate involves inventional embolization [14-15], drug delivery system [16-19], encapsulation of cells [20-23] and cellular therapeutics [24] etc. Recently, researchers are very much interested in Sodium alginate based Casson nanofluids flows. A comprehensive study on the MHD flow of sodium alginate based Casson nanofluid flow through a porous medium with Newtonian heating is done by Khan et al [25]. Alwawi et al [26] studied the MHD natural convection flow of sodium alginate based Casson nanofluid over a solid sphere 
by Killer box method. They analysed the impact of Casson parameter, magnetic parameter, nano particle volume friction parameter on the velocity, temperature and skin friction coefficient. The effects of variable viscosity and thermal conductivity on the Casson nanofluid flow with velocity slip and convective heating is investigated by Gbadeyan et al [27]. A numerical investigation of conjugate heat transfer of water nanofluid flow inside the axisymmetric microchannel is studied by Shahrestani et al [28].

From the motivation of the above study, we want to analyse the nanofluid flow through a porous microchannel under streaming potential effects. The main objective of this investigation is to study the velocity and heat transport for Casson-nano fluid flow in porous microtube under the effect of streaming potential with electrokinetic force associated with constant pressure gradient and applied magnetic field. The analytical solutions of velocity and temperature distribution for the Casson-nano fluid flow are derived using the various dimensionless parameters. The transport phenomenon of this fluid flow has been examined under the combined effect of the electric double layer and applied magnetic field as well as streaming potential effect. The velocity and temperature expression are obtained. Furthermore, the streaming potential and Nusselt number are obtained analytically and investigated the variations of Nusselt number and streaming potential with various non-dimensional parameters graphically.

\section{Mathematical modeling and problem description}

The EMHD flow of a sodium alginate based Casson-nano fluid in a porous microtube under the effect of streaming potential is considered along axial direction of the microtube. The physical sketch of this flow problem is depicted in Fig 1 . This circular microtube is replete by a porous medium where $\mathrm{R}$ and $\mathrm{L}$ denote the radius of the microtube and length of the microtube respectively. The radius of the microtube is much smaller than its length, i.e. $(2 \mathrm{R} \ll \mathrm{L})$. The electrolyte solution generates a EDL near to the boundary wall and the induced electric field in opposite axial direction creates a electroosmotic flow. In the radial direction, the electromagnetic field is induced by the applied magnetic field and electric field. The polar, cylindrical coordinate $(r, \theta, z)$ is considered to describe the governing equations of this flow problem. A constant heat flux $\mathrm{q}_{\mathrm{w}}$ is imposed on the impermeable wall of the microtube. Streaming potential $\mathrm{E}_{\mathrm{s}}$ is generated due to the EDL near the microtube wall which is directed opposite to the fluid motion. The total system is subjected to a applied magnetic field $\mathrm{B}_{0}$ perpendicular to the fluid flow direction and $\mathrm{E}_{1}$ is imposed as an external transverse electric field from outside to inside.

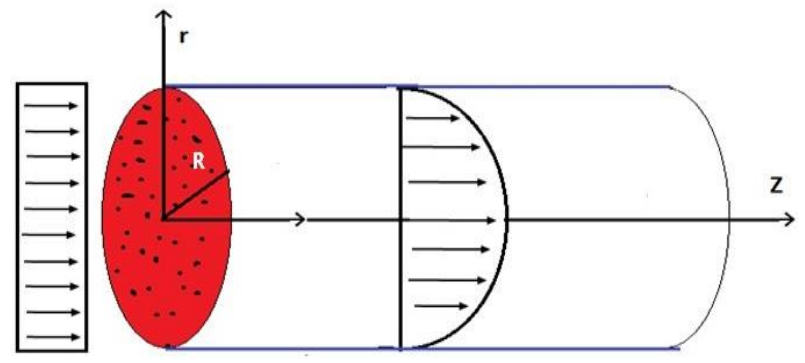

Fig. 1: Schematic Diagram of Circular Microtube with Porous Medium.

\subsection{Governing equations and velocity distribution analysis}

In this flow problem Casson nanofluid is considered where the base fluid is the non-Newtonian Casson fluid. The Casson fluid sample plays Newtonian and non-Newtonian both fluid behavior. The constitutive equation for Casson fluid model can be followed by

$\tau_{i j}=\left\{\begin{array}{l}\left(\mu_{c}+\frac{P_{s}}{\sqrt{2 \pi}}\right) 2 e_{i j}, \pi>\pi_{0} \\ \left(\mu_{c}+\frac{P_{s}}{\sqrt{2 \pi_{0}}}\right) 2 e_{i j}, \pi<\pi_{0}\end{array}\right.$

Where the yield stress of the fluid is $P_{s}=e_{i j} e_{i j}$ and $e_{i j}$ indicates the deformation rate at $(i, j)$ th component. $\pi$ is the product of the component of deformation rate with itself, $\pi_{0}$ is a critical value of this product based on the non-Newtonian fluid model, $\mu_{c}$ represents the plastic dynamic viscosity of the non-Newtonian fluid. Then from (1) it is obtained that (for $\pi<\pi_{0}$ )

$\tau_{\mathrm{ij}}=\mu_{\mathrm{c}}\left(1+\frac{1}{\beta}\right) 2 \mathrm{e}_{\mathrm{ij}}$

where $\beta=\mu_{c} \sqrt{2 \pi_{0}} / P_{s}$ is the casson parameter.

In the present study, Sodium alginate $\left(\mathrm{C}_{6} \mathrm{H}_{9} \mathrm{NaO}_{7}\right)$ is considered as a base fluid. Aluminium oxide $\left(\mathrm{Al}_{2} \mathrm{O}_{3}\right)$ is the nano-particle which is suspended in the base fluid. Then the thermofluidic properties of the nanofluid is as follows:

Table 1: Thermophysical Properties of Nanofluids

\begin{tabular}{cccc}
\hline & $\rho\left(\mathrm{kgm}^{-3}\right)$ & $\mathrm{C}_{\mathrm{P}}\left(\mathrm{kg}^{-1} \mathrm{k}^{-1}\right)$ & $\mathrm{k}\left(\mathrm{Wm}^{-1} \mathrm{k}^{-1}\right)$ \\
\hline $\left.\mathrm{C}_{6} \mathrm{H}_{9} \mathrm{NaO}_{7}\right)$ & 989 & 4175 & 0.613 \\
$\mathrm{Al}_{2} \mathrm{O}_{3}$ & 3970 & 765 & 40 \\
\hline
\end{tabular}

The expressions for effective viscosity and effective density of the nano fluid are given by

$\mu_{\mathrm{eff}}=\frac{\mu_{\mathrm{f}}}{(1-\phi)^{2.5}}, \rho_{\mathrm{eff}}=(1-\phi) \rho_{\mathrm{f}}+\phi \rho_{\mathrm{P}}$

Where the $\mu_{\mathrm{f}}$ is the viscosity of the base fluid, $\rho_{\mathrm{f}}$ and $\rho_{\mathrm{P}}$ represent the densities of the base fluid and the nano particles respectively, $\phi$ is called the volume friction of the nanoparticles. The flow problem is considered as thermally fully developed, steady, symmetrical in azimuthal direction flow under cylindrical co-ordinate system. Therefore, the velocity component is only in $\mathrm{z}$-direction, which depends on $\mathrm{r}$. 
The simplified form of the momentum conservation equation along the z-direction, assuming a hydrodynamically fully developed flow of a Casson nanofluid is written as:

$-\frac{\partial p}{\partial z}+\mu_{\text {eff }}\left(1+\frac{1}{\beta}\right)\left(\frac{d^{2} u}{d^{2}}+\frac{1}{r} \frac{d u}{d r}\right)-\mu_{\text {eff }} \frac{u}{K}+\rho_{e} E_{s}+\sigma_{e} B_{0} E_{1}-\sigma_{e} B_{0}^{2} u=0$

Where $\beta$ is Casson parameter, $\mathrm{K}$ denoted the permeability of the porous medium andE $\mathrm{E}_{0}$ is the axial component and $\mathrm{E}_{1}$ is the lateral transverse component of applied electrical force. Due to small ratio of the diameter and length of the microtube, although the body force may exist in the radial or angular direction and the velocity in these directions is much smaller than the axial direction. Therefore only one momentum equation is considered in the axial direction. The boundary conditions are derived as follows:

$\mathrm{u}(\mathrm{r}=\mathrm{R})=0$ and $\frac{\mathrm{du}}{\mathrm{dr}}(\mathrm{r}=0)=0$

The net charge density $\rho_{\mathrm{e}}$ in the EDL depends on the EDL potential $\psi$ and it is given by [29]:

$\rho_{\mathrm{e}}=-\varepsilon \kappa^{2} \psi$

The EDL potential $\psi$ is well constructed by the Poisson-Boltzmann equation with the assumptions (i) the permittivity of the fluid is not influenced by the field strength and it is constant; (ii) the ions are considered as point charges. Then under the Debye- Hückle approximation the EDL potential distribution is obtained as:

$\psi=\psi_{\mathrm{w}} \mathrm{I}_{0}(\kappa \mathrm{r}) / \mathrm{I}_{0}(\kappa \mathrm{R})$

Where $H$ is the half height of the channel, $\zeta$ be the constant zeta potential and $1 / \kappa$ be the thickness of the EDL where $\kappa=\operatorname{ez}\left(\frac{2 n_{0}}{\varepsilon \mathrm{k}_{\mathrm{B}} \mathrm{T}_{\mathrm{a}}}\right)^{\frac{1}{2}}$ is called Dybye- Hückle parameter where $\mathrm{n}_{0}$ is the ion density (in molar unit) of the liquid, $\mathrm{z}$ is the valance of ions of the solution, $\mathrm{k}_{\mathrm{B}}$ is the Boltzmann constant, and $\mathrm{T}_{\mathrm{a}}$ is the absolute temperature, $\varepsilon$ is permittivity of the fluid and $\omega=\kappa \mathrm{R}$ is called the normalized reciprocal thickness of the EDL which representing the ratio of half height of microchannel to Debye length (i.e. $1 / \kappa)$.

Let us introduce the following dimensionless variables and parameters:

$u^{*}=\frac{u}{u_{H S}}, r^{*}=\frac{r}{R}, G=\frac{\left(-\frac{\partial p}{\partial z}\right) R^{2}}{\mu_{f} u_{H S}}, \psi^{*}=\psi\left(\frac{e z}{k_{B} T}\right), \psi_{w}^{*}=\psi_{w}\left(\frac{e z}{k_{B} T}\right)$,

$E_{S}^{*}=\frac{E_{S}}{E_{0}}, D a=\frac{K}{R^{2}}, H a=B_{0} R \sqrt{\frac{\sigma_{e}}{\mu_{f}}}, u_{H S}=\frac{-\varepsilon E_{0} k_{B} T}{e z \mu_{f}}, S=\frac{E_{1} R}{u_{H S}} \sqrt{\frac{\sigma_{e}}{\mu_{f}}}$

Where $u_{H S}$ be the reference velocity, $G$ be the non-dimentional pressure gradient. $H a$ is the Hartmann number which is indicating the strength of the applied magnetic field $B_{0}$ and $D a$ is the Darcy number. $S$ represents the strength of the transverse electric $E_{1}$ field. The nondimensional form of the equation (13) is reduced by:

$\frac{\left(1+\frac{1}{\beta}\right)}{(1-\phi)^{2.5}}\left(\frac{d^{2} u^{*}}{d r^{* 2}}+\frac{1}{r^{*}} \frac{d u^{*}}{d r^{*}}\right)+\omega E_{S}^{*} \psi^{*}-\left(H a^{2}+\frac{1}{D a(1-\phi)^{2.5}}\right) u^{*}+H a S-G=0$

Boundary condition (5) is reduced as follows:

$u^{*}\left(r^{*}=1\right)=0$ and $\frac{d u^{*}}{d r^{*}}\left(r^{*}=0\right)=0$

Equation (8) is analytically solved analytically based the boundary conditions (9) to obtain the exact solutions which is written as

$u^{*}\left(r^{*}\right)=\frac{I_{0}\left(M r^{*}\right)}{I_{0}(M)}\left\{\frac{A E_{s}^{*} M^{2}}{M^{2}-\omega^{2}} I_{0}(\omega)+B\right\}-B-\frac{A E_{s}^{*} M^{2}}{M^{2}-\omega^{2}} I_{0}\left(\omega r^{*}\right)$

where $A=\frac{(1-\phi)^{2.5} \omega^{2} \psi_{w}^{*}}{(1+1 / \beta) I_{0}(\omega)}, B=\frac{(G-H a S)(1-\phi)^{2.5}}{(1+1 / \beta)}, M=\frac{\left\{(1 / D a)+H a^{2}(1-\phi)^{2.5}\right\}}{(1+1 / \beta)}$

The above expression of velocity function depends on different physical parameters like Hartmann number $\mathrm{Ha}$, Casson parameter $\beta$, Darcy number $D a$, volume fraction parameter $\phi$ etc.

\subsection{Streaming potential}

The streaming potential is obtained from the following expression:

$i_{\text {net }}=I_{S t}+I_{C o}=0$

Which tells that the net ionic current through the tube section equal to zero. Where $I_{S t}$ is Streaming current and $I_{C o}$ is the Conduction current. It is assumed that the ions are moved within the EDL in the electrolyte solution. Then the streaming current and Conduction current are written as:

$I_{S t}=2 \pi \int_{0}^{R} z e\left(n_{+}-n_{-}\right) u r d r$ 
$I_{C o}=2 \pi \int_{0}^{R} z e\left(n_{+}+n_{-}\right)\left(\frac{e z E_{S}}{f}\right) r d r$

Here $f=2 n_{0} e^{2} z^{2} / \sigma_{b}$ is called the ionic friction factor where $\sigma_{b}$ is the bulk ionic conductivity and $n_{ \pm}=n_{0} e x p\left(\mp \frac{e z \psi}{k_{B} T_{a v}}\right)$ is the ionic number concentration of positively and negatively charge species. By substituting all the dimensionless variables, the following dimensionless form is obtained:

$\int_{0}^{1} \psi^{*} u^{*} r^{*} d r^{*}-\alpha^{*} E_{S}^{*} \int_{0}^{1} r^{*} d r^{*}=0$

Where $\alpha^{*}=\mu \sigma_{b} / 2 n_{0} \varepsilon k_{B} T_{a v}$ represents the non-dimensional conductivity parameter. And finally the non-dimensional streaming potential is given by

$$
E_{S}^{*}=\frac{2 \tau_{1}^{*}}{\alpha^{*}-2 \tau_{2}^{*}}
$$

Here $\tau_{1}^{*}$ and $\tau_{2}^{*}$ is obtained from the following expressions:

$$
\begin{aligned}
& \tau_{1}^{*}=\int_{0}^{1} \frac{\psi_{w}^{*} A I_{0}\left(\omega r^{*}\right)}{I_{0}(\omega)}\left\{\frac{I_{0}(\omega) I_{0}\left(M r^{*}\right)}{\left(M^{2}-\omega^{2}\right) I_{0}(M)}-\frac{M^{2} I_{0}\left(\omega r^{*}\right)}{\left(M^{2}-\omega^{2}\right)}\right\} r^{*} d r^{*} \\
& \tau_{2}^{*}=\int_{0}^{1} \frac{\psi_{w}^{*} B I_{0}\left(\omega r^{*}\right)}{I_{0}(\omega)}\left\{\frac{I_{0}\left(M r^{*}\right)}{I_{0}(M)}-1\right\} r^{*} d r^{*}
\end{aligned}
$$

\subsection{Temperature distribution and heat transfer analysis}

The governing equations of energy equation for thermally fully developed flow is expressed as

$\left(\rho C_{P}\right)_{e f f} u \frac{\partial T}{\partial z}=k_{e f f}\left\{\frac{1}{r} \frac{\partial}{\partial r}\left(r \frac{\partial T}{\partial r}\right)+\frac{\partial^{2} T}{\partial z^{2}}\right\}+\Omega+S_{j}$

where $\left(\rho C_{P}\right)_{\text {eff }}$ is the effective heat capacitance of the nano-fluid and which is calculated by the following expression:

$$
\left(\rho C_{P}\right)_{e f f}=\phi\left(\rho C_{P}\right)_{P}+(1-\phi)\left(\rho C_{P}\right)_{f}
$$

Where $\left(\rho C_{P}\right)_{P}$ and $\left(\rho C_{P}\right)_{f}$ are called the heat capacities per unit volume of the solid nano particles and base fluid respectively. Here $\Omega=$ $\mu_{e f f}\left\{\left(1+\frac{1}{\beta}\right)\left(\frac{\partial u}{\partial r}\right)^{2}+\frac{u^{2}}{K}\right\}, S_{j}=\sigma_{e}\left(E_{s}^{2}+B_{0}^{2} u^{2}-2 E_{1} B_{0} u\right) . \Omega$ indicates the combination of viscous dissipation and the dissipation due to the Darcy term, $S_{j}$ is the volumetric heat generation due to joule heating respectively. Where, $T$ is the local temperature of the liquid. and $k_{e f f}$ is the thermal conductivity of the nano-fluid which is calculated by the following expression:

$k_{e f f}=k_{f}\left(\frac{k_{p}+2 k_{f}-2\left(k_{f}-k_{p}\right) \phi}{k_{p}+2 k_{f}+2\left(k_{f}-k_{p}\right) \phi}\right)$

Where $k_{p}$ is thermal conductivity of the nano particles and $k_{f}$ is the thermal conductivity of the base fluid. Introducing the non-dimensional temperature $T^{*}=\frac{k_{f}\left(T-T_{w}\right)}{q_{w} R}$ where $T_{w}$ is the channel wall temperature and $q_{w}$ is the constant wall heat flux. Further, for thermally fully developed flow under imposed constant wall heat flux, one may write $\frac{\partial T}{\partial z}=\frac{d T_{w}}{d z}=\frac{d T_{b}}{d z}=$ constant and $\frac{d^{2} T}{d z^{2}}=0$ in which $T_{b}$ is the bulk mean temperature. The overall energy balance of an elementary control volume of the fluid with the length of duct $d x$ gives the following expression:

$$
\left(\rho C_{P}\right)_{e f f} \pi R^{2} u_{m} \frac{d T_{b}}{d z}=2 \pi R q_{w}+2 \pi \int_{0}^{R} \Omega r d r+\sigma_{e}\left\{E_{S}^{2} \pi R^{2}+\int_{0}^{R}\left(B_{0}^{2} u^{2}-2 E_{1} B_{0} u\right) r d r\right\}
$$

Then the bulk mean temperature gradient can be obtained as

$$
\frac{d T_{b}}{d z}=\frac{\gamma_{1}}{\left(\rho C_{P}\right)_{e f f}}=\text { constant }
$$

where $\gamma_{1}=\frac{1}{R u_{m}}\left\{2 q_{w}+\sigma_{e} E_{S}^{2} R+\frac{2 \mu_{\text {eff }}}{R}\left\{\left(1+\frac{1}{\beta}\right) \beta_{2}+\frac{\beta_{1}}{K}\right\}+\frac{\sigma_{e} B_{0}^{2} \beta_{1}}{R}\right\}-\sigma_{e} E_{1} B_{0} R$,

$\beta_{1}=\int_{0}^{R} u^{2} r d r, \beta_{2}=\int_{0}^{R}\left(\frac{d u}{d r}\right)^{2} r d r$ and $u_{m}=\frac{\int_{0}^{2 \pi} \int_{0}^{R} u r d r d \theta}{\pi R^{2}}$, which is called axial mainstream velocity. The following dimensionless parameters are introduced to make dimensionless the equation (16)

$\gamma=\left(\frac{\gamma_{1} u_{H S} R}{q_{w}}\right), B r=\frac{\mu u_{H S}}{h q_{w}}, S_{J}=\frac{\sigma_{e} E_{S}^{2} R}{q_{w}}$

where $\gamma$ is the ratio of the heat generated by the interaction of the electric and magnetic fields to heat conduction, $B r$ is the Brinkman number which describes the ratio of heat produced by viscous dissipation and heat transport by molecular conduction, $S_{J}$ is the joule heating due to heat conduction. Then by using (18) the non-dimensional form of the equation (16) is obtained as 
$\frac{k_{e f f}}{k_{f}}\left\{\frac{1}{r^{*}} \frac{\partial}{\partial r^{*}}\left(r^{*} \frac{\partial T^{*}}{\partial r^{*}}\right)\right\}=\gamma u^{*}-B r\left\{\frac{\left(1+\frac{1}{\beta}\right)}{(1-\phi)^{2.5}}\left(\frac{d u^{*}}{d r^{*}}\right)^{2}+\left(\frac{D a}{(1-\phi)^{2.5}}+H a^{2}\right) u^{* 2}-2 H a S u^{*}\right\}-S_{J}$

The corresponding non-dimensional boundary conditions are expressed as

$T^{*}(-1)=0, \frac{d T^{*}(0)}{d y^{*}}=0$

Since the temperature equation (21) is a non-linear differential equation, thus it is solved numerically with the corresponding boundary conditions (22). By applying the fourth order Runge-Kutta method one can solve the equations numerically using the shooting technique in MATLAB.

According to the obtained temperature distribution and velocity distribution, the non-dimensional bulk temperature can be written by the following expression:

$\widetilde{\theta^{*}}=\frac{\int_{0}^{2 \pi} \int_{0}^{1} u^{*} T^{*} r^{*} d r^{*} d \theta}{\int_{0}^{2 \pi} \int_{0}^{1} u^{*} r^{*} d r^{*} d \theta}=\frac{k_{e f f}\left(T_{w}-T_{m}\right)}{R q_{w}}$

In thermal transport phenomenon an important heat transfer parameter can be expressed as Nusselt number $\mathrm{Nu}$, which illustrates the rate of heat transfer and can be defined as:

$$
N u=\frac{2 R q_{w}}{k_{e f f}\left(T_{w}-T_{m}\right)}=-\frac{k_{f}}{k_{e f f}} \frac{2}{\vec{\theta}^{*}}
$$

\section{Results and discussion}

In this case study, microfluidic transport of Sodium alginate based Casson nanofluid is considered. It is well known that sodium alginate is a natural Casson fluid ; also it is hydrophilic, highly viscous gum. For Casson nanofluid, Aluminium oxide $\left(\mathrm{Al}_{2} \mathrm{O}_{3}\right)$ is taken as nanoparticles with sodium alginate. This section deals with the investigation of the influences of various pertinent parameters on the fluid flow, heat transfer. The obtained results of the velocity and temperature distribution are discussed in this section. Further, the geometrical interpretation of streaming potential and rate of heat transfer are also illustrated. The values ([9],[10] and [13]) of the parameters $(\omega=4, H a=$ $\left.0.5, D a=0.02, G=0.5, \psi_{w}^{*}=-1, \alpha^{*}=0.1, \phi=0.05, \beta=0.5, S=50, B r=0.01, S_{J}=0.6\right)$ are kept unchanged throughout the study unless otherwise states.

(A)

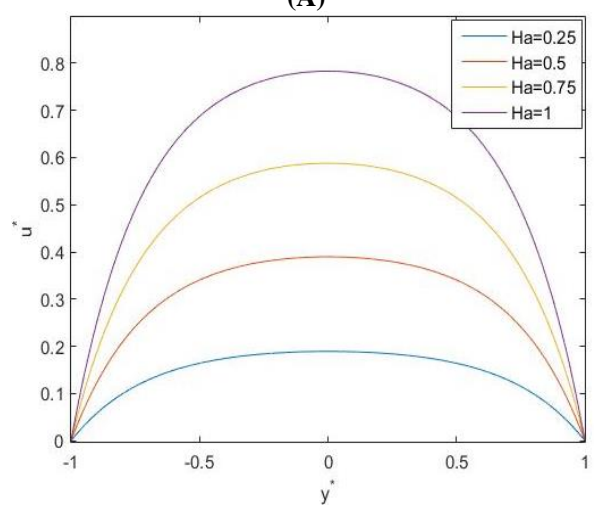

(C)

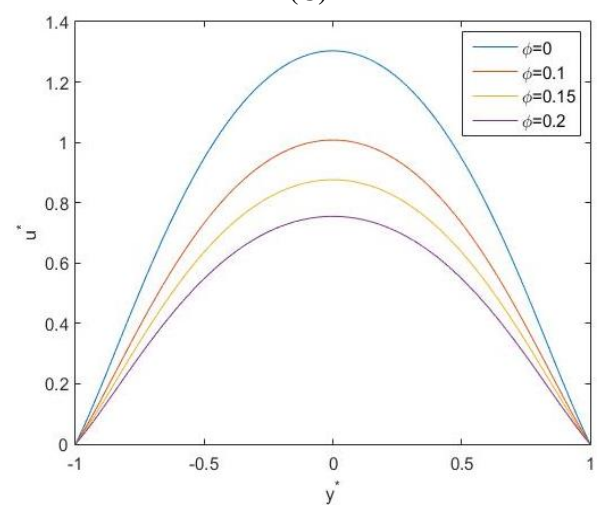

(B)

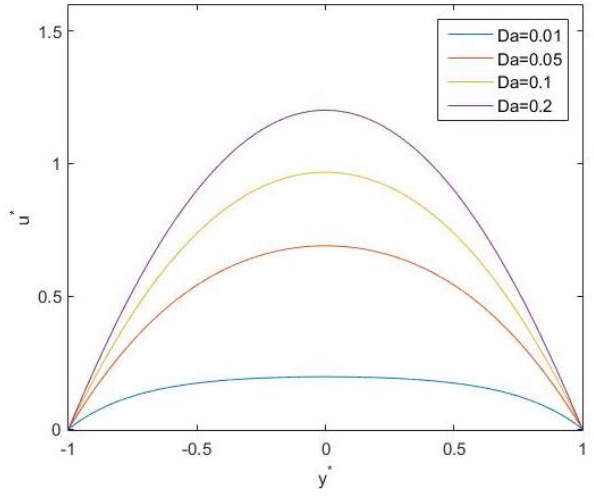

(D)

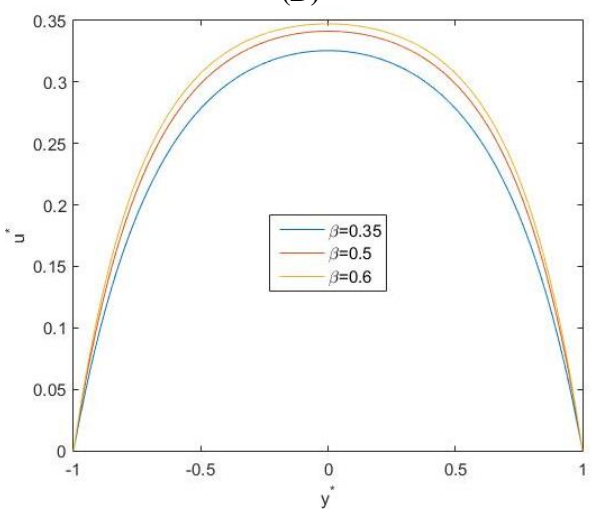

Fig. 2: Velocity Distribution Plots with Variation of (A) Hartmann Number (B) Darcy Number (C) Nano-Particle Volume Friction (D) Casson Parameter.

The velocity and temperature profiles are represented in figure 2 and 3. It can be seen that the velocity distribution have the parabolic velocity profile and the velocity is maximum in the middle layers of the flow in the channel. When $\boldsymbol{S}=\mathbf{5 0}$, the velocity profile have the increasing trend with the increases of the applied magnetic field and the increases of the Darcy number which are illustrated in figure 2(a) and 2(b) respectively. It can be observed that from equation (8) the electromagnetic force is a combination of the linear variation of transverse electric field $\left(\boldsymbol{E}_{\mathbf{1}}\right)$ and the suare of the magnetic field $\left(\boldsymbol{B}_{\mathbf{0}}\right)$. Thus, there subsist two adding forces acting on the fluid flow motion, 
the component of pressure gradient and the component of electromagnetic force $\left(\boldsymbol{\sigma}_{e} \boldsymbol{B}_{\mathbf{0}} \boldsymbol{E}_{\mathbf{1}}\right)$ and $\left(\boldsymbol{\sigma}_{\boldsymbol{e}} \boldsymbol{B}_{\mathbf{0}}^{\mathbf{2}} \boldsymbol{u}\right)$ acting as a retardind force. Hence due to the presence of the transverse electric field i.e. for the flow adding force the fluid velocity is enhanced. The velocity is slower for low Darcy number. But in the presence of transverse electric field the velocity profile is depreciated with the increase values of the nano particle volume friction which is shown in figure 2(c). It can be demonstrated from the figure 2(c) that when $\boldsymbol{\phi}=\mathbf{0}$, then the velocity is maximum and the velocity becomes slow for large value of nano particle volume friction parameter. The reason behind this phenomenon is that the effective viscosity is increases with the enhancement of the volume friction parameter in response to shear rate which depreciated the fluid flow motion. Also it can be observed from figure 2(d) that the velocity has increasing trend with the increases of Casson parameter.

(A)

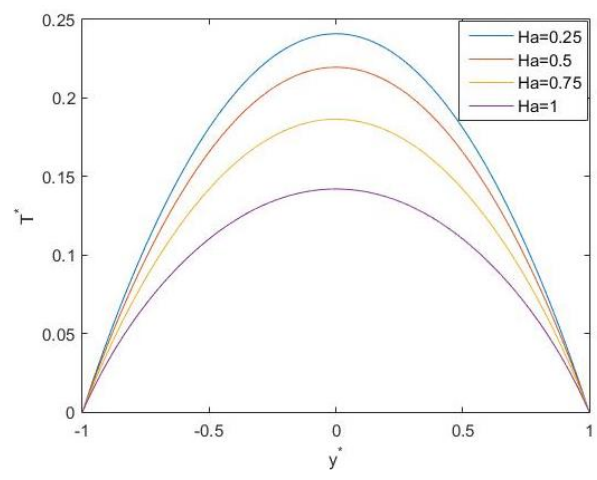

(C)

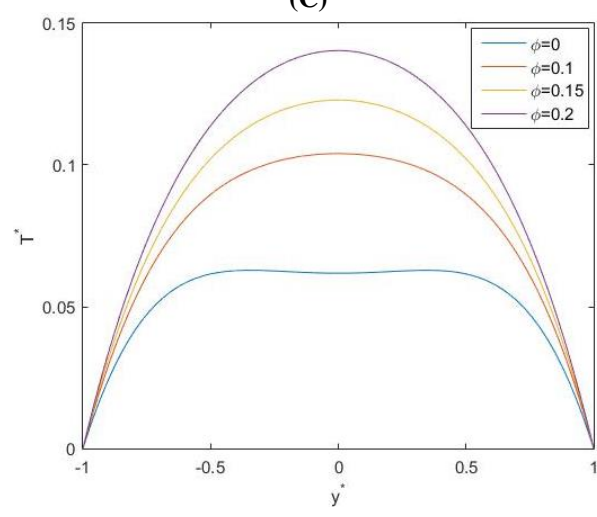

(E)

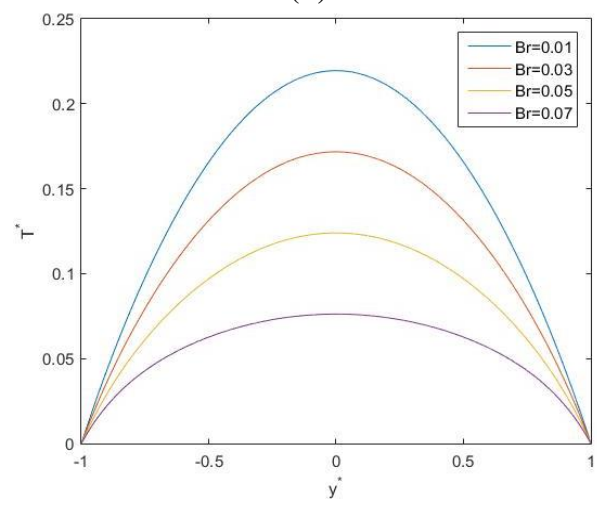

(B)

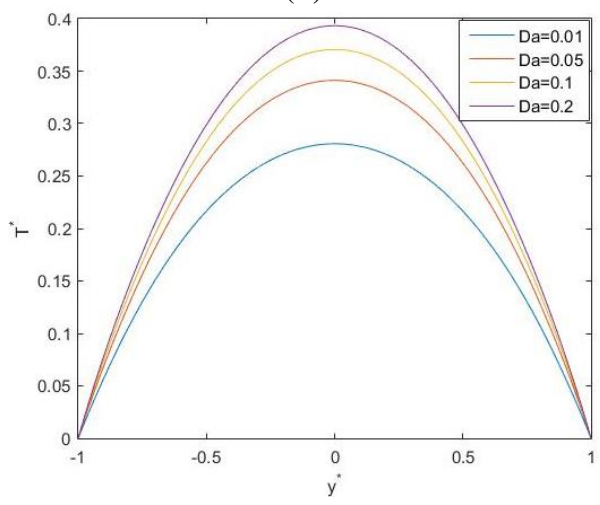

(D)

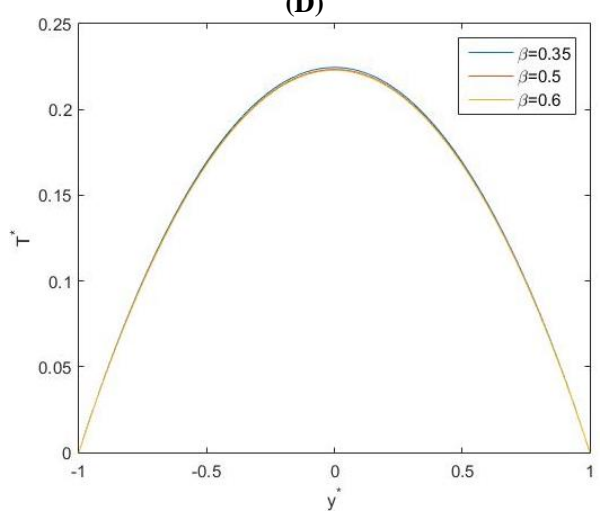

(F)

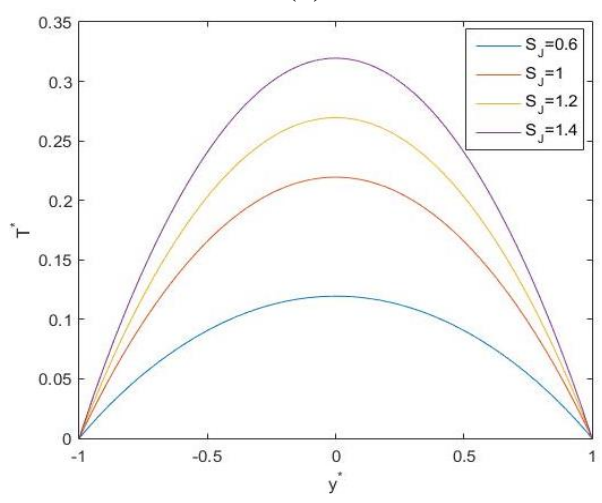

Fig. 3: Temperature Distribution Plots with Variation of (A) Hartmann Number (B) Darcy Number (C) Nano-Particle Volume Friction (D) Casson Parameter (E) Brinkmann Number (F) Joule Heating Parameter.

The geometrical approach of the temperature distribution is delineated in figure 3. It is demonstrated in figure 3(a) that the temperature profile decreases with the increasing trend of the applied magnetic field. Also, when the Darcy number is increasing then the temperature is decreasing which is displayed in the figure 3(b). Figure 3(c) delineated that the temperature profile is decreased with increases of the nano particle volume friction. It is failed to trigger any apprehensible change in the associated temperature profile with the increasing trend of the Casson parameter which is represented in figure 3(d). The temperature is maximum at the middle layers of the flow. The increment of Brinkmann number depreciated the temperature profile which is shown in figure 3(e). The temperature becomes low for large value of Brinkmann number. Form figure 3(f), it is depicted that the temperature profile has an enhancement with the increment of the joule heating parameter. 
(A)

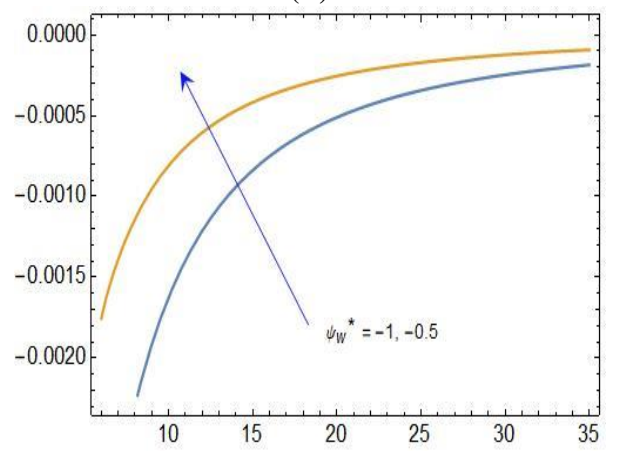

(B)

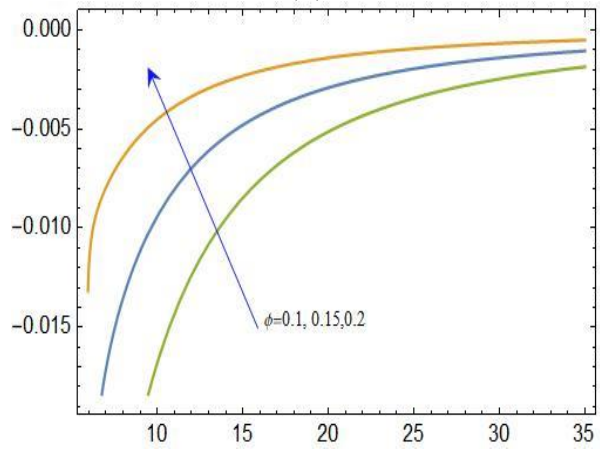

Fig. 4: Streaming Potential (Y-Axis) Plot with $\omega$ (X-Axis) (A) with Different Wall Zeta Potential (B) with Different Values of the Nano-Particle Parameter.

The variation of dimensionless streaming potential with the non-dimensional EDL thickness are ilustrated in figure 4(a) and 4(b) for different dimensionless wall zeta potential and nano-particle volume friction parameter respectively. It can be observed that the dimensionless streaming potential exhibits the abatement with the increment of the dimensionless EDL thickness parameter and the magnitude of the streaming potential ultimately tending to zero.

The variation of Nusselt number with other pertinent parameters are displayed in figure 5 . The Nusselt number is decreased gradually with the enhancement of the applied magnetic field and increases slowly for the higher value of the volume friction parameter of the nanoparticles which is depicted in figure 5(a). From the figure 5(b), it is depicted that the Nusselt number is decreased swiftly for lower Darcy number but after a certain limit it is increased gradually with the increases of the Darcy number. However, for the higher value of the nanoparticle parameter, the rate of heat transfer increases slowly. It may note from the figure 5(c) that the Nusselt number is decreased gradually with the enhancement of the transverse electric field and increases slowly for the higher value of the volume friction parameter of the nanoparticles. The Nusselt number increases rapidly for an increasing trend of the joule heating parameter within the low range and the impact of the nano particle volume friction parameter on the Nusselt number are not very comprehensive with joule heating parameter which is illustrated in figure $5(\mathrm{~d})$.

(A)

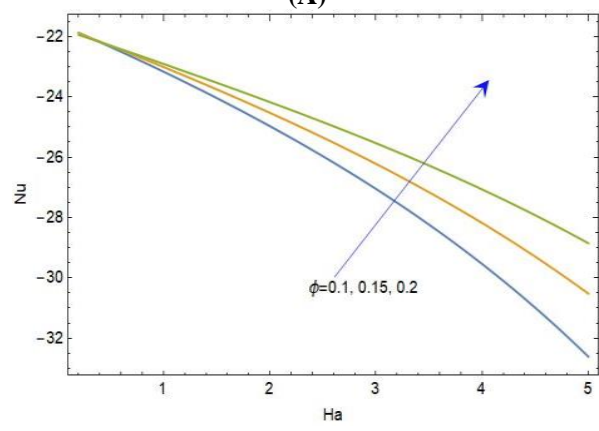

(C)

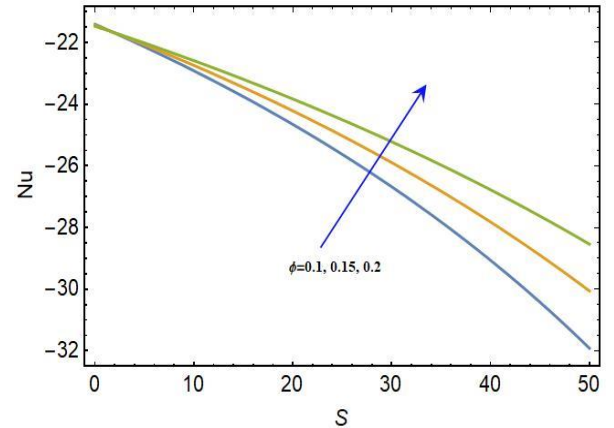

(B)

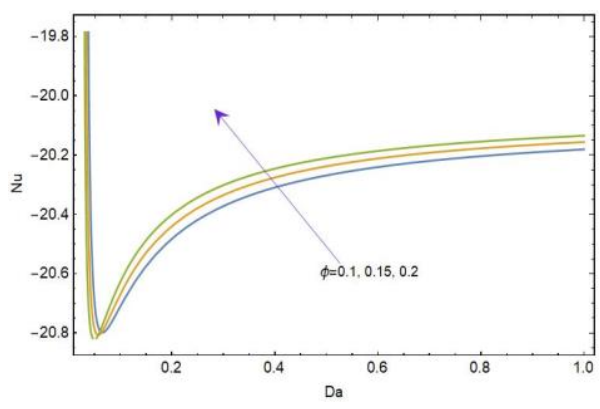

(D)

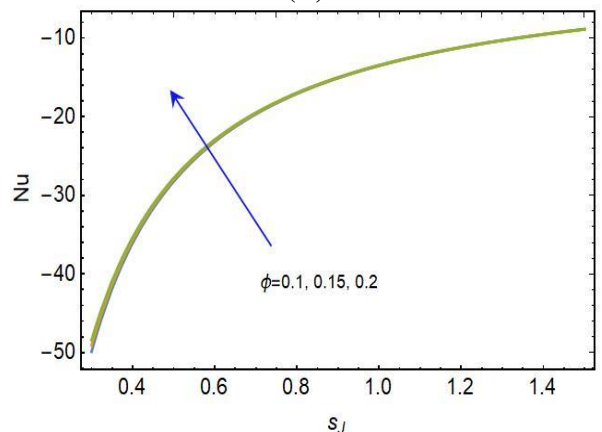

Fig. 5: Effects of Nano Particle Volume Friction on Nusselt Number with (A) Hartmann Number, (B) Darcy Number, (C)Varying Magnitude of Transverse Electric Field, (D) Joule Heating Parameter.

\section{Conclusions}

In this present study, the velocity and temperature distribution is analysed numerically for the EMHD flow of Casson-nanofluid through a porous microchannel under the streaming potential effects. The following observations can be drawn from this numerical study:

- The velocity is proportional to the nano particle volume friction but the temperature profile is inversely proportional to the nano particle volume friction.

- The velocity and temperature both are always maximum in the middle layers of the flow.

- The streaming potential is inversely proportional to the dimensionless EDL thickness parameter.

- The Nusselt number has an improvement with the increases of the volume friction parameter with the other pertinent parameter. 


\section{Acknowledgement}

This work was supported by SERB, Govt of India (Grant File No. EMR/2016/006383). The authors would like to acknowledge this support.

\section{References}

[1] H.A. Stone, A.D. Stroock, A. Ajdari, Engineerring flows in small devices: Microfluidics toward a Lab-on-a-chip, Annual Review of Fluid Mechanics 36 (2004) 381-411. https://doi.org/10.1146/annurev.fluid.36.050802.122124.

[2] T. Bayraktar, S.B. Pidugu, Characterization of liquid flows in microfluidic systems, International Journal of Heat and Mass Transfer 49 (2006) 381 411. https://doi.org/10.1016/j.ijheatmasstransfer.2005.11.007.

[3] A. Behnampour, O. A. Akbari, M. R. Safaei, M. Ghavami, A. Marzban, G. A. S. Shabani, M. zarringhalam, R. Mashayekhi., Analysis of heat transfer and nanofluid fluid flow in microchannels with trapezoidal, rectangular and triangular shaped ribs. Physica E: Low-dimensional Systems and Nanostructures (2017) S1386-9477 (17) 30281-3.

[4] J. Li, C. Kleinstreuer, Thermal performance of nanofluid flow in microchannels, International Journal of Heat and Fluid Flow 29, 4, (2008), 12211232 https://doi.org/10.1016/j.ijheatfluidflow.2008.01.005.

[5] J. Yang, J. H. Masliyah, and D. Y. Kwok, Streaming Potential and Electroosmotic Flow in Heterogeneous Circular Microchannels with Nonuniform Zeta Potentials: Requirements of Flow Rate and Current Continuities., Langmuir, (2004), 20, 3863-3871 https://doi.org/10.1021/la035243u.

[6] L. Gong, J. Wu, L. Wang, and K. Cao, Streaming potential and electroviscous effects in periodical pressure-driven microchannel flow, Physics of Fluids, (2008) 20, 063603. https://doi.org/10.1063/1.2939391.

[7] F. Lu, J. Yang, and D. Y. Kwok, Flow Field Effect on Electric Double Layer during Streaming Potential Measurements, J. Phys. Chem. B (2004), 108, 14970-14975. https://doi.org/10.1021/jp048277z.

[8] S. Chakraborty, D. Paul. Microchannel flow control through a combined electromagnetohydrodynamic transport. J Phys D: Appl Phys; 39: (2006) 5364-71. https://doi.org/10.1088/0022-3727/39/24/038.

[9] R. Chakraborty, R. Dey, S. Chakraborty.Thermal characteristics of electromagnetohydrodynamic flows in narrow channels with viscous dissipation and Joule heating under constant wall heat flux. International Journal of Heat and Mass Transfer ,67, (2013), 1151-1162. https://doi.org/10.1016/j.ijheatmasstransfer.2013.08.099.

[10] M. Reza, A. Rana, Analysis of Exact Solutions of Electromagnetohydrodynamic Flow and Heat Transfer of Non-Newtonian Casson Fluid in Microchannel with Viscous Dissipation and Joule Heating, Advances in Fluid Mechanics and Solid Mechanics, (2020), DOI:10.1007/978-981-15-07724_11 https://doi.org/10.1007/978-981-15-0772-4_11.

[11] M. Buren,Y. Jian and Chang L; Electromagnetohydrodynamic ow through a microparallel channel with corrugated walls J. Phys. D: Appl. Phys. 47; (2014), 425-501. https://doi.org/10.1088/0022-3727/47/42/425501.

[12] M. Rashid, I. Shahzadi, S. Nadeem. Corrugated walls analysis in microchannels through porous medium under Electromagnetohydrodynamic (EMHD) effects, Results in Physics 9; (2018), 171-182. https://doi.org/10.1016/j.rinp.2018.02.023.

[13] G. Zhao, Y. Jian, F. Li. Heat transfer of nanofluids in microtubes under the effects of streaming potential, Applied Thermal Engineering 100; (2016), 1299-1307. https://doi.org/10.1016/j.applthermaleng.2016.02.101.

[14] Q. Wang, K. Qian, S.S. Liu, Y.J. Yang, B. Liang, C.S. Zheng, X.L. Yang, H.B. Xu, A.Q.Shen, X-ray visible and uniform alginate microspheres loaded with in situsynthesized BaSO4nanoparticles for in vivo transcatheter arterialembolization, Biomacromolecules 16 (2015) $1240-1246$. https://doi.org/10.1021/acs.biomac.5b00027.

[15] T.A. Becker, D.R. Kipke, T. Brandon, Calcium alginate Gel: a biocompatible andmechanically stable polymer for endovascular embolization, J. Biomed. Mater.Res. 54 (2001) 76-86. https://doi.org/10.1002/1097-4636(200101)54:1<76::AID-JBM9>3.0.CO;2-V.

[16] C.D.M.P. Matricardi, T. Coviello, F. Alhaique, Recent advances and perspectiveson coated alginate microspheres for modified drug delivery, Expert Opin.Drug Deliv. 5 (2008) 417-425. https://doi.org/10.1517/17425247.5.4.417.

[17] H.H. Tønnesen, J. Karlsen, Alginate in drug delivery systems, Drug Dev. Ind.Pharm. 28 (2002) 621-630. https://doi.org/10.1081/DDC-120003853.

[18] T.K. Giri, D. Thakur, A. Alexander, A. Ajazuddin, H. Badwaik, D.K. Tripathi, Alginate based hydrogel as a potential biopolymeric carrier for drug deliveryand cell delivery systems: present status and applications, Cur. Drug Deliv. 9(2012) 539-555. https://doi.org/10.2174/156720112803529800.

[19] Y. Hori, A.M. Winans, D.J. Irvine, Modular Injectable matrices based onalginate solution/microsphere mixtures that gel in situ and co-deliverimmunomodulatory factors, Acta Biomater. 5 (2009) 969-982 https://doi.org/10.1016/j.actbio.2008.11.019.

[20] S. Akbari, T. Pirbodaghi, Microfluidic encapsulation of cells in alginateparticles via an improved internal gelation approach, Microfluid. Nanofluid.16 (2013) 773-777 https://doi.org/10.1007/s10404-013-1264-Z

[21] H. Zimmermann, S.G. Shirley, U. Zimmermann, Alginate-based encapsulationof cells: past, present, and future, Curr. Diabetes Rep. 7 (2007) 314 320. https://doi.org/10.1007/s11892-007-0051-1.

[22] C.J. Martinez, J.W. Kim, C. Ye, I. Ortiz, A.C. Rowat, M. Marquez, D.A. Weitz,Microfluidic approach to encapsulate living cells in uniform alginate hydrogelmicroparticles, Macromol. Biosci. 12 (2012) 946-951. https://doi.org/10.1002/mabi.201100351.

[23] L.B. Zhao, L. Pan, K. Zhang, S.S. Guo, W. Liu, Y. Wang, Y. Chen, X.Z. Zhao, H.L.Chan, Generation of Janus alginate hydrogel particles with magneticanisotropy for cell encapsulation, Lab Chip 9 (2009) 2981-2986. https://doi.org/10.1039/b907478c.

[24] B.P. Barnett, A. Arepally, M. Stuber, D.R. Arifin, D.L. Kraitchman, J.W.M. Bulte, Synthesis of magnetic resonance-, X-ray- and ultrasound-visible alginatemicrocapsules for immunoisolation and noninvasive imaging of cellulartherapeutics, Nat. Protoc. 6 (2011) 1142-1151. https://doi.org/10.1038/nprot.2011.352.

[25] Arshad Khan, Dolat Khan, Ilyas Khan, Farhad Ali, Faizan ul Karim, Muhammad Imran, MHD Flow of Sodium Alginate-Based Casson Type NanofluidPassing Through A Porous Medium with Newtonian Heating, Scientific Reports (2018) 8:8645, https://doi.org/10.1038/s41598-01826994-1.

[26] Firas A. Alwawi, Hamzeh T. Alkasasbeh, A.M. Rashad, Ruwaidiah Idris, MHD natural convection of Sodium Alginate Casson nanofluid over a solid sphere, Results in Physics 16 (2020) 102818. https://doi.org/10.1016/j.rinp.2019.102818.

[27] J.A.Gbadeyan, E.O.Titiloye, A.T.Adeosun, Effect of variable thermal conductivity and viscosity on Casson nanofluid flow with convective heating and velocity slip, Heliyon 6 (2020) e03076. https://doi.org/10.1016/j.heliyon.2019.e03076.

[28] Misagh Irandoost Shahrestani, Akbar Maleki, Mostafa Safdari Shadloo andIskander Tlili, Numerical Investigation of Forced Convective Heat Transfer and Performance Evaluation Criterion of $\mathrm{Al}_{2} \mathrm{O}_{3}$ /Water Nanofluid Flow inside an Axisymmetric Microchannel, Symmetry 2020, 12, 120; https://doi.org/10.3390/sym12010120.

[29] J.H. Masliyah and S. Bhattacharjee. Electrokinetic and Colloid Transport Phenomena (Hoboken, NJ: Wiley-Interscience) (2006). https://doi.org/10.1002/0471799742. 